(Aus dem Kaiserin Auguste Vietoria-Haus zur Bekämpfung der Säuglings: sterblichkeit im Deutsehen Reiche [Dir. Prof. Dr. L. Langstein].)

\title{
Das Vorkommen und die klinische Bedeutung der eosinophilen Zellen im Säuglingsalter, besonders bei der exsudativen Diathese.
}

\author{
Von \\ Dr. Hermann Putzig, \\ Assistent.
}

Mit I Textfigur.

(Eingegangen am 30. Oktober 1913.)

Mit der Prüfung des Vorkommens und der klinischen Physiologie und Pathologie der eosinophilen Leukocyten speziell im Säuglings- und frühesten Kindesalter wird ein Gebiet betreten, das schon zahlreiche Untersucher bearbeitet haben.

Doch hat mir eine genaue Durchsicht der erreichbaren einschlägigen Literatur gezeigt, daß zwischen den verschiedenen Untersuchern schon in Beziehung auf die Normalwerte große Differenzen bestehen. Die Gründe dafür liegen, wie es scheint, teilweise in der angewandten Methode, die, wie ich später zeigen will, große Fehlerquellen hat, teilweise in der nicht hinreichend präzisen Fassung des Begriffes ,normaler, gesunder Singling"

Es schien mir also wichtig, zunächst einwandfreie Normalwerte zu schaffen, um dann auf Grund dieser bei gesunden Säuglingen mit guter Methode gefundenen Zahlen Schlüsse aus den Ergebnissen bei kranken Säuglingen zu ziehen.

\section{Physiologisches.}

Zunächst sollen an dieser Stelle aus der Literatur über die Eosinophilen und ihre Bedeutung kurz die wichtigsten Daten folgen. Die eosinophilen Leukocyten wurden als besondere Zellform von Ehrlich erkannt, der ihre färberischen und biologischen Eigenschaften feststellte. Utber die Herk unft der Eosinophilen sind auch heute noch die Ansichten. 
geteilt. Ehrlich nahm für sie, wie für die übrigen Leukocyten, als Ursprungsort das Knochenmark an, eine Ansicht, die er später dahin erweiterte, daß er eine Entstehung aus den fixen Bindegewebszellen als möglich hinstellte. Dieser Ansicht, die von einem großen Teil der Forscher anerkannt wird, steht die andere gegenüber, die eine lokale Entstehung annimmt (s. Pappenheim).

Auch über die Entstehungsart ist man nicht einig. Ehrlich nahm an, daß. die Granula Produkte des Zellkernes sind (s. a. Löwit), andere Forscher glauben, daß die Granula aus aufgenommenen roten Blutkörperchen teils direkt (Goldzieher, Weidenreich, Klein, Petry), teils nach Umwandlung (Spuler und Schittenhelm) gebildet werden. Diese Punkte sowie die chemische Zusammensetzung der eosinophilen Granula seien hier nur gestreift. Ausführliche Zusammenstellungen finden sich bei Bettmann, K. Meyer, Stäubli, Hertzell, Pappenheim sowie in den Lehrbüchern der Hämatologie (Türk, Naegeli).

Gehen wir nun zu den klinischen Ergebnissen über, so ergibt sich folgendes:

Der Durchschnittsnormalwert beim Erwachsenen, wie ihn schon Ehrlich festgestellt hat, steht mit 2-4\% der Gesamtzah] der Leukocyten ziemlich fest, wenn auch $G$ ala m bos in letzter Zeit mit der später zu erwähnenden Dungerschen Methode auch beim normalen Menschen schwankende Werte von $0,48-10,5 \%$ gefunden hat. Gala mbos weist darauf hin, daß Schwankungen um 100\% bei demselben Individuum durchaus nicht zu den Seltenheiten gehören. Ähnliche Befunde hat schon früher Zappert festgestellt. Auf Grund seiner Untersuchungen möchte Gala mbos dem Vorhandensein von eosinophilen Zellen jegliche diagnostische Bedeutung absprechen. Von anderer Seite (Gelbart aus der Sahlischen Klinik) ist dagegen ihr diagnostischer und prognostischer Wert aufs neue hervorgehoben worden.

Im folgenden sind die Krankheiten zusammengestellt, bei denen sich eine Vermehrung der Eosinophilen ergab:

1. Tierparasiten (Ebell, Herrik, Jaffa, Low, Naegeli, Pappenheim, Weinberg, Whyte);

2. Gewisse Hautaffektionen, besonders idiopathisches Ekzem, Urticaria, Arzneiexantheme (Naegeli, Türk, Neußer, Langstein, Pappenheim, Stäubli, Bruck, Peter, Wolf-Eisner);

3. Parenterale Eiweißzufuhr (Schlecht, Schwenker und Schlecht, Neußer, Ahl und Schittenhelm); 
4. Anaphylaxie (Schwenker und Schlecht, Ahl und Schittenhelm, Wolfsohn u. a.);

5. Frühjahrs - Katarrh (Herbert, Steiger und Strebel);

6. Asthma (Heinecke und Deutschmann, Galambos, Langstein, Jaffa, Naegeli, Rosenstern);

7. Psychosen, Neurosen, Vagotonie (Zappert, K. Meyer, Türk, Naegeli, Neußer, Pappenheim).

8. Scharlach (Naegeli, Türk, Stäubli u. a.);

9. Postinfektiös, posttoxisch, posthämorrhagisch (Arneth, Naegeli, Coskinas, Leuret et Gauvenet).

Verminderung der Eosinophilen finden wir:

1. Bei Infektionskrankheiten (Arneth, Gelbart, Loos, Ebell, Ehrlich, K. Meyer, Schwarz, Galambos, Gundobin, Meunier);

2. Bei schweren Verdauungstörungen (Longa, Weiß, Benjamin, Zelensky);

3. bei chronischen und letalen Wurmkrankheiten (Low, Hertzell);

4. bei Adrenalin- sowie Salz-Injektionen (Gaisböck, Schlecht und Schwenker, Skorzewski und Wasserberg, Rosenstern).

Zweifelhaft waren die Resultate, d. h. ergaben teils normale Werte, teils Schwankungen zwischen Verminderung und Vermehrung, bei

1. Erkrankungen der Drüsen mit in nerer Sekretion - Diabetes, Akromegalie usw. - (Caro, Huguenin, Mendel, Mennacher, K. Meyer, Borchardt, Eggers, Bahrdt und Benfey, Kahler);

2. Tuberkulose und Lues (Galambos, Hirschfeld, Loos, Grawitz, NeuBer, S(holz);

3. Blutkrankheiten (Ehrlich, Schwarze, Neußer, Longa, Luzet, Zelensky, Mosse - Grünbaum, Müller und Rieder).

Wir wollen uns nunmehr zu unserem Spezialgebiet wenden, d. h. zu der Frage nach dem Vorkommen und der Bedeutung der Eosinophilen im Kindes-, besonders im Säuglingsalter. Auch hier sind die Meinungen geteilt und zwar sowohl über die Physiologie als auch über die Pathologie. Die Frage hat besonders an Interesse gewonnen, nachdem festgestellt wurde, daß verschiedene, zum Symptomenkomplex der exsudativen Diathese (Czerny) gehörige Affektionen von einer Vermehrung der Eosinophilen begleitet sind. Schon früher sind allerdings zahlreiche, speziell das Kindesalter betreffende Arbeiten erschienen, die 
sich insbesondere mit der Frage der diagnostischen Bedeutung der eosinophilen Zellen bei den verschiedenen Blutkrankrankheiten des Kindes beschäftigen, nachdem Ehrlich den Eosinophilen eine differential-diagnostisch wichtige Rolle bei der Erkennung und Unterscheidung von Leukämie und Pseudo-Leukämie zugewiesen hatte. Später wurden dann auch andere Krankheiten untersucht, besonders die Rachitis, Tuberkulose, Lues, Infektionskrankheiten, Ernährungsstörungen (s. auch die obige Zusammenstellung).

Einwandfreie Resultate ergaben sich nur für die akuten Infektionen und Ernährungsstörungen (Verminderung), Scharlach und gewisse Hautkrankheiten (Vermehrung), während für die Rachitis (Aschenheim, Finday, Hook und Schlesinger, Loos u. a.) sowie für die Blutkrankheiten sich nichts Sicheres feststellen ließ.

Für die exsudative Diathese, sowohl für Ekzem wie Darmerscheinungen, hat, soweit sich aus der Literatur ersehen läßt, als erster Langstein auf die Vermehrung der Eosinophilen hingewiesen. Allerdings hat schon vorher Neußer und besonders Peters u. a. auf die hohe Zahl von Eosinophilen beim Prurigo und anderen zum sogenannten Arthritismus gehörigen Affektionen aufmerksam gemacht.

Es hat sich dann im Laufe der Zeit ein Streit erhoben über die Frage, inwieweit diese Zellen für die Diathese als solche typisch sind und inwieweit sie nur von der Hautaffektion bei der Diathese abhängen. Darauf soll später eingegangen werden.

An dieser Stelle muß, besonders auch um die Differenzen zwischen den einzelnen Untersuchern zu erklären, darauf hingewiesen werden, daß, wie oben erwähnt, nicht einmal die Frage nach dem normalen Gehalt des kindlichen Blutes an eosinophilen Zellen ausreichend geklärt ist. Während nämlich auf der einen Seite behauptet wurde, daß die Zahl dieser Zellen relativ dieselbe sei, wie im Blute der Erwachsenen, wurde von anderen Untersuchern eine auffallend hohe Zahl von Eosinophilen im Säuglingsblute festgestellt (s. darüber die spätere Tabelle). Je nachdem nun aber die Normalwerte, die die verschiedenen Untersucher als gegeben annahmen, schwankten, mußten natürlich auch die Schlüsse, die. sich auf diese Befunde stützten, verschieden ausfallen.

Was bisher an Normalzahlen vorliegt, kann in verschiedener Hinsicht. nicht als unanfechtbar gelten. Teilweise ist die $\mathrm{Zahl}$ der untersuchten Kinder zu klein, um weitere Schlüsse zu erlauben, teilweise ist der Begriff ,,gesunder Säugling" nicht scharf genug abgegrenzt, indem ihn die Untersucher nur auf den Zustand des Kindes zur Zeit der Unter- 
suchung bezogen, nicht aber, wie dies Rosenstern zum ersten Male erkannt und durchgeführt hat, auf Kinder, die in langer Beobachtungszeit als gesund befunden wurden. Manchmal sind sogar eigentlich kranke Kinder mitgerechnet (z. B. Zivcovic). Besonders aber ist die Technik der Untersuchung in fast allen bisherigen Arbeiten mit ganz wenigen Ausnahmen (Karnitzki) sehr anfechtbar. Es wurde nämlich bisher in allen Fällen die Zahl der eosinophilen Zellen im Ausstrichpräparat bestimmt. Nun ist aber, wie bekannt, die Verteilung der Zellen im Ausstrich, selbst bei größter Übung, nicht gleichmäßig, und nur ein Auszählen von 1000 Zellen und mehr gewährleitet einigermaßen gute Resultate, während das Auszählen von 200, ja selbst 500 Zellen, Fehlermöglichkeiten bietet, die bei dem relativ geringen Gehalt des Blutes an Eosinophilen bedeutsam sein müssen.

\section{Eigene Untersuchungen.}

Technik.

Es war also nötig, bei der Bestimmung der Normalwerte mit besonderer Vorsicht ans Werk zu gehen.

Als Material für die Untersuchung stand mir die Abteilung für gesunde Säuglinge im Kaiserin Auguste Victoria-Haus zur Verfügung. Es war hier Gelegenheit geboten, im Hause selbst geborene Kinder wochen- und monatelang in jeder Beziehung ärztlich zu beobachten und alle die Kinder auszuschalten, bei denen sich irgendwelche Krankheitserscheinungen darboten. Die dem Haus angegliederte Fürsorge machte ein weiteres Verfolgen der Fälle auch nach der Entlassung zum größten Teil ein ganzes Jahr hindurch möglich.

Als Methode für die Untersuchung benutzte ich nicht wie die früheren Forscher die Zählung im Ausstrichpräparat, sondern die Kammerfärbung nach Dunger, die gegenüber anderen Kammerfärbungen (Zollikofer, Schueffner, Elzholtz und Lenzmann), die gerade die Eosinophilen schlecht färben, den Vorteil hat, daß sie alle anderen Zellen zerstört und $n$ ur die Eosinophilen färbt. Die Methode, die auch von Galambos und Gelbart, welche sie, wie erwähnt, bei Erwachsenen anwendeten, sehr empfohlen wird, gewährleistet, wie ich durch Vergleich mit Ausstrichpräparaten feststellen konnte, eine weit größere Genauigkeit als das Auszählen von 1000 Zellen im Ausstrich. Bei der Auszählung von $4 \times 9$ großen Quadraten der Buerkerschen Kammer werden mehr Zellen gezählt, als bei der Auszählung von 2000 Zellen im Ausstrich. Ubrigens ist die Mischung des 
434 H. Putzig: Vorkommen und klinische Bedeutung der eosinophilen Zellen

Tabelle I, Untersuchungt

\begin{tabular}{|c|c|c|c|c|c|c|c|c|c|c|c|c|c|c|c|c|c|c|c|c|}
\hline \multirow[b]{2}{*}{ Nr. } & \multicolumn{3}{|c|}{ 1. Woche } & \multicolumn{3}{|c|}{ 2. Woche } & \multicolumn{3}{|c|}{ 3. Woche } & \multicolumn{3}{|c|}{ 4. Worhe } & \multicolumn{3}{|c|}{ 2. Monat } & \multicolumn{3}{|c|}{ 3. Monat } & \multicolumn{2}{|c|}{ 4. Monat } \\
\hline & 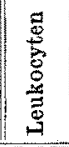 & $\begin{array}{c}\mathrm{E} 0 \\
\mathrm{n} \\
\mathrm{ph} \\
\mathrm{o} \\
\mathrm{E}\end{array}$ & $\%$ & 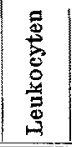 & $\begin{array}{c}10 \\
\mathrm{n} \\
\mathrm{ph} \\
0 \\
\frac{0}{\sigma} \\
\end{array}$ & $\%$ & 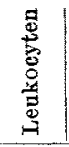 & $\begin{array}{c}\mathbf{E} 0 \\
\mathrm{n} \\
\mathrm{ph} \\
\mathrm{j} \\
\mathrm{a}\end{array}$ & $\begin{array}{l}\text { si. } \\
0- \\
\text { ile } \\
\%\end{array}$ & 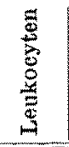 & $\begin{array}{c}\text { Eo } \\
n \\
\text { ph } \\
0 \\
0 \\
\end{array}$ & $\begin{array}{l}\text { si- } \\
\text { o- } \\
\text { iile } \\
\%\end{array}$ & 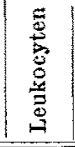 & $\begin{array}{c}\text { Eo } \\
n \\
p h \\
\dot{\infty} \\
\frac{\dot{a}}{\omega}\end{array}$ & $\begin{array}{c}\text { si- } \\
0= \\
\text { ile } \\
\%\end{array}$ & 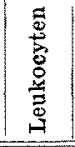 & $\begin{array}{c}\mathrm{Eo} \\
\mathrm{no} \\
\mathrm{ph} \\
\dot{0} \\
\stackrel{0}{\Xi}\end{array}$ & $\begin{array}{c}0.1 \\
0- \\
\text { ile } \\
\%\end{array}$ & 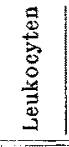 & 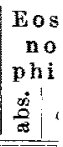 \\
\hline 1. 1 & 660 & 100 & 1,06 & - & -1 & & 12660 & 132 & & - & $1-1$ & $\rightarrow$ & 400 & $|60|$ & $\mid 0,58\}$ & - & $=$ & $1-$ & 10400 & \\
\hline 2. & 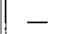 & - & - & - & - & - & - & - & & - & - & - & 10200 & 92 & $0,90 \mid$ & - & - & - & - & - \\
\hline 3. $\mathrm{F}$ & - & - & - & - & - & - & 11500 & 317 & 2,7 & - & - & -1 & 11000 & 211 & 1,91 & $|10700|$ & 188 & 1,71 & - & - \\
\hline 4. & 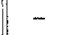 & - & -7 & - & -1 & $1-1$ & - & - & - & - & - & -1 & 9600 & $100 \mid$ & 1,04 & 9470 & $65 \mid \mathrm{C}$ & 0 & - & - \\
\hline 5. $\mathrm{B}$ & - & - & - & 9800 & 206 & 2,1 & - & - & - & -. & -1 & - & - & - & - & 8500 & 133 & 1,57 & - & \\
\hline 6. & 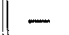 & - & - & - & - & - & 7900 & 225 & $2,8 \tilde{a}$ & - & - & -1 & 7800 & 198 & 2,54 & - & - & - & - & $1-$ \\
\hline 7. $\mathrm{H}$ & & - & - & 11300 & 291 & $2, \mathbf{5 8} \mid$ & - & -1 & - & - & $-\infty$ & - & - & -1 & - & 8480 & {$[2 ; 0]$} & 2,95 & - & - \\
\hline 8. $\mathrm{Fr}$ & 9000 & 286 & 8,18 & - & - & $1-$ & - & -1 & - & - & -1 & - & 9100 & 183 & $2,01\}$ & - & -1 & -1 & 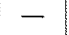 & - \\
\hline 9. $\mathrm{En}$ & - & - & - & 10800 & 212 & 1,97 & - & - & - & - & - & - & 9100 & 175 & 1,92 & - & - & - & - & - \\
\hline s & - & - & - & - & - & - & 7900 & 156 & 1,97 & - & -1 & - & - & - & $1-$ & 8300 & 206 & 2,48 & - & - \\
\hline $\mathrm{B}$ & - & - & - & 9700 & 126 & 1,30 & - & -1 & - & - & - & - & 8200 & 83 & 1,01 & 7800 & 95 & 1,22 & - & - \\
\hline $\mathrm{F}$ & & -1 & - & -1 & - & & - & - & - & 8700 & $|100|$ & 1,13 & - & -1 & - & 8500 & 120 & 1,41 & - & - \\
\hline $\mathrm{x}$ & - & - & - & - & - & $1-$ & - & - & - & 8900 & 189 & 2,12 & 9800 & 156 & 1,59 & - & - & - & - & - \\
\hline .8 & - & - & - & - & - & - & - & $1-$ & - & - & - & - & 7600 & $|172|$ & 2,26 & - & - & - & - & - \\
\hline & & -1 & - & - & - & - & - & $1-$ & - & 8100 & 183 & 2,26 & - & - & - & 7900 & 195 & 2,47 & - & - \\
\hline 3. $\mathrm{K}$ & - & - & - & - & - & - & 11900 & 245 & 2,06 & - & - & - & 10500 & 178 & 1,70 & - & - & - & - & - \\
\hline $\mathrm{I}$ & - & - & - & - & - & - & - & - & - & 10600 & $\lfloor 16$ & 1,09 & - & - & - & - & -1 & - & 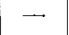 & - \\
\hline & & - & - & - & - & - & - & - & - & 8100 & 158 & $|1,9 \mathbf{s}|$ & - & - & - & - & -1 & - & - & \\
\hline s & & - & - & - & - & - & 14000 & 144 & 1,0 & - & - & $1-1$ & 12100 & 156 & 1,28 & 3) - & -1 & $1-$ & - & - \\
\hline 6 & 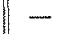 & - & $1-$ & 一 & - & - & 12700 & 238 & 1,87 & - & -1 & -1 & 10800 & 214 & 1,98 & | & $1-1$ & $1-$ & - & - \\
\hline 8 & - & - & - & - & $1-1$ & $1-$ & 7600 & 125 & 1,64 & - & -1 & - & - & - & - & 8100 & 154 & 1,9 & - & - \\
\hline & & - & - & 10000 & 2886 & 2,86 & - & - & -1 & - & -1 & - & - & - & -1 & - & -1 & - & 6900 & 65 \\
\hline . $M$ & - & - & - & - & $1-$ & - & - & - & - & 8200 & 68 & $0,88\}$ & - & $1-$ & -1 & - & -1 & -1 & - & - \\
\hline 4. Kl. & - & 1 & - & - & $1-$ & - & 8900 & 250 & 2,81 & - & $1-1$ & $1-$ & - & $1-$ & - & - & $1-1$ & -1 & 7600 & 10 \\
\hline & & & & & & & & & & & & 1,04 & 97 & & 1,5 & 864 & 10 & 1,8 & oen & $\mid 151\}$ \\
\hline
\end{tabular}

Gesamtresultat: berechneter Durchschnitt: 8890 Leukocyte Stellungsmittel: 8700 Leukocyte

Blutes eine weit exaktere, als bei Deckglas- oder gar Objektträger-Präparaten. Die Zählung ist auBerdem sehr bequem, da bei der $\mathrm{D} u \mathrm{ngerschen}$ Färbung eben nur die Eosinophilen gefärbt werden, so daß die Auszählung von $4 \times 9$ Quadraten der Buerkerschen Kammer, die ich benutzte, bei einiger Ubung nicht mehr als 10 Minuten in Anspruch nimmt.

Zur Bestimmung der relativen Eosinophilenwerte wurden in einer zweiten Mischpipette die Leukocyten mittels T uer kscher Lösung gefärbt und ebenfalls in der Buerkerschen Kammer gezählt.

Als Ort der Blutentnahme wählte ich die Ferse, die vorher mit Alkohol und Äther gereinigt war, als Zeit fast immer $21 / 2-3$ Stunden nach dem Anlegen bzw. der Mahlzeit.

Ich habe die Kinder in vier Kategorien geteilt. 
bei gesunden Säuglingen.

\begin{tabular}{|c|c|c|c|c|c|c|c|c|c|c|c|c|c|c|c|c|c|c|c|c|c|c|c|}
\hline \multirow[b]{2}{*}{ 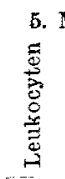 } & \multicolumn{2}{|c|}{ Monat } & \multicolumn{3}{|c|}{ 6. Monat } & \multicolumn{3}{|c|}{ 7. Monat } & \multicolumn{3}{|c|}{ 8. Monat } & \multicolumn{3}{|c|}{ 9. Monat } & \multicolumn{3}{|c|}{ 10. Monat } & \multicolumn{3}{|c|}{ 11. Monat } & \multicolumn{3}{|c|}{ 12. Monat } \\
\hline & $\mid \begin{array}{c}\mathrm{E} 0 \\
\mathrm{n} \\
\mathrm{ph} \\
\dot{\infty} \\
\frac{\dot{g}}{\infty}\end{array}$ & $\begin{array}{l}\text { si- } \\
0 . \\
\text { ile } \\
0\end{array}$ & 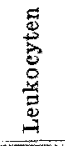 & $\begin{array}{r}\text { En } \\
n \\
1 \mathrm{~h} \\
\dot{\theta} \\
\frac{\dot{\theta}}{\infty}\end{array}$ & $\begin{array}{l}\text { si- } \\
0- \\
\text { ile }\end{array}$ & 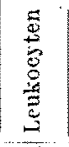 & $\mid \begin{array}{c}F_{0} \\
n \\
p h \\
\dot{\theta} \\
\frac{B}{\infty}\end{array}$ & $\begin{array}{c}\text { sl- } \\
0- \\
\text { ille } \\
y \\
y\end{array}$ & $\begin{array}{l}5 \\
8 \\
3 \\
3 \\
8 \\
0 \\
3 \\
3 \\
3 \\
3\end{array}$ & $\mid \begin{array}{r}\mathrm{Eo} \\
\mathbf{n} \\
\mathrm{ph} \\
\mathrm{d} \\
\mathrm{g}\end{array}$ & $\begin{array}{l}3 \mathrm{si}- \\
0 \\
11 \mathrm{e} \\
0 \\
0\end{array}$ & 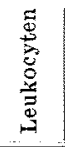 & $\begin{array}{r}\mathrm{Eo} \\
\mathrm{n} \\
\mathrm{ph} \\
\mathrm{d} \\
\mathrm{B}\end{array}$ & & 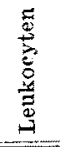 & \begin{tabular}{r}
$n$ \\
$\mathrm{ph}$ \\
$\stackrel{0}{\mathrm{~g}}$ \\
\multirow{3}{*}{}
\end{tabular} & e & 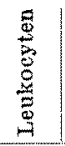 & 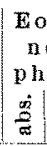 & $\begin{array}{c}\text { si. } \\
0- \\
\text { ille } \\
\% \\
\%\end{array}$ & 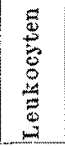 & $\mid \begin{array}{c}\mathrm{No} \\
\mathrm{n} \\
\mathrm{p} \\
0 \\
0 \\
0 \\
\mathrm{O}\end{array}$ & $\begin{array}{l}\text { i } \\
\text { ie } \\
\text { i }\end{array}$ \\
\hline 800 & & 0,7 & 100 & 90 & 1,11 & 7800 & 105 & 1,30 & - & - &..- & 一 & - & - & 7900 & 112 & 1,42 & - & - & $1-$ & 7300 & tho & 1,62 \\
\hline 060 & 8 & 0,86 & - & - & - & - & - & - & - & - & - & 一 & - & - & - & - & -1 & 8500 & $\infty$ & 1,06 & - & - & - \\
\hline- & - & $\ldots$ & 9600 & 169 & 1,76 & $\ldots$ & $\ldots$ & - & - & - & - & - & $1-$ & - & - & - & - & - & $=$ & - & - & - & - \\
\hline 8700 & 83 & 0,95 & 一 & - & $1-5$ & - & - & - & - & - & - & 一 & - & - & - & - & - & - & - & - & 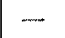 & - & - \\
\hline- & - & - & - & - & - & - & $1 \ldots$ & - & - & - & - & - & - & - & - & - & - & 7600 & 105 & 1,38 & $\ldots$ & - & $\ldots$ \\
\hline - & - & -.. & - & - & - & - & - & - & - & - & - & - & - & - & - & $\ldots$ & - & - & - & - & $\ldots$ & - & - \\
\hline- & - & - & - & - & - & - & - & - & - & - & - & - & - & - & - & - & - & - & - & - & - & - & - \\
\hline - & - & - & - & - & 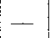 & - & - & - & - & - & & - & - & - & - & - & - & - & - & - & - & - & $\therefore$ \\
\hline 300 & 178 & 2,11 & - & - & - & - & - & - & - & - & - & 一 & - & - & - & - & - & - & $=$ & - & - & - & - \\
\hline- & - & -1 & 7300 & 183 & 2,51 & - & - & - & - & - & - & - & - & - & - & $=$ & - & - & - & - & - & - & - \\
\hline 7900 & 118 & 1,49 & 一 & - & 1 & - & - & - & - & - & - & - & - & $\ldots$ & - & - & - & - & - & - & - & - & - \\
\hline- & - & 13 & - & - & $1-$ & - & - & - & - & $\ldots$ & ـ & - & $\ldots$ & $1-$ & $\ldots$ & - & 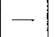 & - & - & - & - & - & - \\
\hline - & - & - & - & - & - & - & - & - & -- & - & - & - & - & - & - & - & - & - & - & - & $\ldots$ & - & - \\
\hline 3300 & 154 & 1,8 & - & - & 一 & - & - &..- & - & - & - & - & $-\infty$ & - & - & - & - & - & - & $1-$ & - & - & - \\
\hline- & - & - & 一 & - & - & - & - & - & 7600 & 217 & 2,86 & 一 & - & - & - & - & - & - & - & - & - & - & - \\
\hline$\ldots$ & - & - & - & - & - & - & - & - & - & - & 12 & - & -2 & - & - & - & & - & - & - & - & - & - \\
\hline 3900 & 275 & 3,20 & - & - & - & - & - & 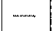 & - & - & $\ldots$ & - & - & - & - & - & - & - & - & - & - & - & - \\
\hline 9100 & 21 & 2,31 & - & - & - & 8300 & 195 & 2,35 & - & - & - & 8600 & 184 & 2,14 & - & $1-\ldots$ & - & - & - & - & - & - & - \\
\hline- & - & - & - & - & - & - & - & - & - & - & $1-$ & - & $\ldots$ & - & - & - & $\ldots$ & - & $\ldots$ & - & $\ldots$ & - & $=$ \\
\hline- & - & - & - & - & - & - & - & - & $\ldots$ & - & 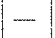 & - & - & - & - & - & - & - & - & - & - & - & \\
\hline- & - & - & - & - & - & - & - & - & - & - &.- & - & - & - & - & - & - & - & - & - & - & - & - \\
\hline- & - & - & - & - & - & 7200 & 85 & 1,18 & - & - & - & - & - & - & - & 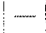 & - & - & - & - & - & - & - \\
\hline 8100 & 85 & 1,05 & - & - & - & - & - & - & $\ldots$ & - & - & - & - & - & - & $=$ & 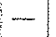 & - & $=$ & - & - & - & - \\
\hline- & $1-$ & - & - & - & - & - & $1-$ & $=$ & - & - & . & 一 & - & - & - & - & - & - & $1-$ & - & - & - & - \\
\hline 560 & & 60 & 30 & & & 70 & 8 & 65 & 00 & $21 \%$ & 86 & 8600 & 184 & 14 & 100 & 11 & 1,42 & 50 & 98 & 1,22 & 300 & 1 & $x$, \\
\hline
\end{tabular}

158 Eosinophile $=1,78 \%$,

$15 \%$ Eosinophile $=\mathbf{1 , 8 0} \%$.

1. Gesunde Säuglinge, die während der ganzen, durch Monate fortgesetzten Beobachtung keine Zeichen einer krankhaften Störung zeigten;

2. sonst normale Säuglinge, bei denen sich im Laufe der Beobachtung Ekzeme, wenn auch nur vorübergehend, zeigten;

3. kranke Säuglinge, mit Ausnahme der Kinder mit exsudativer Diathese;

4. Säuglinge mit Symptomen von exsudativer Diathese.

Die Ergebnisse wurden in Tabellenform gebracht.

\section{Ergebnisse.}

Die Resultate der ersten Gruppe ,gesunde Säuglinge" waren folgende (s. auch Tabelle I): 
Die absoluten Werte schwankten zwischen 60 und 317 Zellen pro cmm Blut, die relativen zwischen 0,58 und 3,20 , der berech nete Durchschnitt war 8890 Leukocyten, 158 Eosinophile $=1,78 \%$. Als Stellungsmittel ergaben sich 8700 Leukocyten, 157 Eosinophile $=1,80 \%$.

Zum Vergleiche mit den bisherigen Resultaten habe ich die Ergebnisse früherer Untersucher and meine eigenen in beifolgender Tabelle II

Tabelle II. Normalwerte der eosinophilen Zellen beim Säugling.

\begin{tabular}{|c|c|}
\hline Aschenheim.... & bis $9,3 \%$ \\
\hline Benjamin..... & $7,4 \%$ (Durehsehnittswert) \\
\hline Bettmann..... & vermehrt \\
\hline Biffi et Galli ... . & $0,5-2,8 \%$ (erste 14 Tago $=0,5-9,0 \%$ ) \\
\hline Canon ..... & $0-14 \%$ \\
\hline Carstanjen .... & $0,35-9,35 \%$ (1.-6. Mon.), $0-2,85 \%$ (6.-12. Mon., \\
\hline . . . . . & $1-2 \%$ \\
\hline Ehrlich . . . . . & vermehrt (normal $2-4 \%$ ) \\
\hline . . . . . . & $1-2,5 \%$ Neugeborene \\
\hline Fischl. . . . . & $0,73-8,61 \%(4,13 \%$ Durchschnittswert $)$ \\
\hline Gundobin I.... & $0,5-7,2 \%$ im ersten Monat, später $1,2 \%$ \\
\hline Gundobin II. . . . & $0,5-5 \%(3 \%$ Durchschnitt $)$ \\
\hline Hock und Schlesinger & $0,5-11 \%$ \\
\hline Jaksch. . . . . . & bis $10 \%$ \\
\hline Japha....... & $7,4 \%$ (Durchschnittswert) \\
\hline Karnitzki..... & $0,9-5,5 \%$ (Durchschnitt $2,5--3,9 \%$ ) \\
\hline Kassowitz..... & vermehrt \\
\hline Langstein..... & bis $1 \%$ \\
\hline K. Meyer . . . . & vermehrt (normal $0,5-4 \%$ ) \\
\hline Müller und Rieder. & $0,39-6,1 \%$ (Neugeborene) \\
\hline Naegeli...... & vermehrt \\
\hline D'Orlandi & $2 \%$ (Neugeborene) \\
\hline Peter....... & $0,9-4,62 \%(1,93 \%$ im Durchsehnitt $)$ \\
\hline Rieder...... & $1-7 \%$ \\
\hline Rosenstern .... & $0,7-4 \%$ \\
\hline Sahli. ...... & vermehrt \\
\hline Stäbli ...... & vermehrt \\
\hline Strshelbitzki ... & bis $25 \%$ \\
\hline Takasu ...... & $0-9,62 \%(2,8 \%$ Durehsohnitt $)$ \\
\hline Türk. . . . . . & vermehrt \\
\hline Weiß... • . . . • & $1,-5,6 \%$ \\
\hline Wolff. . . . . & $1-2 \%$ \\
\hline Zappert...... & $3,46-10,12 \%$ \\
\hline Eigene Ergebnisse: & $\begin{array}{l}0,58-3,20 \% \text {, berechneter Durchschnitt } 158= \\
\text { Stellungsmittel } 157=1,80 \% .\end{array}$ \\
\hline
\end{tabular}


gegenübergestellt. Diese Gegenüberstellung zeigt, daß meine Resultate am meisten den Ergebnissen von Rosenstern und Karnitzki nahekommen, d.h. denjenigen Autoren, die besonders die eingangs aufgestellten Postulate für verwertbare Ergebnisse bei ihren Untersuchungen berücksichtigt haben.

Wie die hohen Werte einzelner Autoren, wie Benjamin u. a., deren Feststellungen dann wiederum zum Ausgang weiterer Betrachtungen dienten, zustande gekommen sind, entzieht sich natürlich der Nachprüfung. Jedenfalls haben meine an einwandfreiem Material erhaltenen Resultate auch für den gesunden Säugling keine anderen Zahlen ergeben, als sie im allgemeinen für den gesunden Erwachsenen angenommen werden. Diese Tatsache ist für die folgenden Betrachtungen besonders wichtig.

Wir kommen nunmehr zu einer zweiten Gruppe, nämlich den Säuglingen, bei denen sich im Laufe der Beobachtung leichtere oder schwerere Hauterscheinungen zeigten.

Die Einzelergebnisse sind in Tabelle III zusammengestellt, aus der auch die Art der Hauterscheinung zu ersehen ist. Es zeigt sich, daß bei Kindern, die später Milchschorf oder Prurigo bekamen, die Werte besonders in der 2.-4. Woche, also zu ciner Zeit, wo sich die Umwandlung vom Blutbild des Neugeborenen in das des Säuglings vollzieht, fast sämtlich oberhalb der Maximalwerte bei gesunden Kindern liegen. Dagegen sind die Werte bei Kindern, die später nur Intertrigo ohne andere rein exsudative Erscheinungen darboten, nicht gegen die Norm erhöht. Auf die Bedeutung dieser Befunde wird später im Zusammenhang mit den Ergebnissen bei exsudativer Diathese näher einzugehen sein.

Ich komme nunmehr zu den kranken Säuglingen, bei denen die eosinophilen Zellen gezählt wurden, und lasse zunächst kurze Auszüge aus den Krankengeschichten folgen:

1. Kind B., früher chronische Verdauungsstörung, Ekzem, öfters Husten. 25. XI. 1912. Lingua geographica, Stomatitis. 5725000 rote Blutkörperchen, 10230 weiße, 245 Eosinophile $=2,39 \%$.

28. XI. 23870 weiße, 71 Eosinophile $=0,3 \%$.

1. XII. Typische Kopliks, beginnendes Masernexanthem im Gesicht, 11500 Leukocyten, 35 Eosinophile $=0,3 \%$.

2. Kind $Z$. Schwere chronische Verdauungsstörung vom Typus Herterscher Infantilis mus. 19.XI.1912. 6860 Leukocyten, 50 Eosinophile $=0,85_{\%}^{\circ}$.

3. I. 1913. 15700 Leukocyten, 145 Eosinophile $=0,92^{\circ}$; im übrigen starke Verminderung der roten Blutkörperchen (2900 000) und des Hämoglobingehalts (24\% nach Sahli). Das Blutbild zeigte außerdem ausgesprochene Mononucleose mit besonderer Vermehrung der großen mononucleären Zellen. 


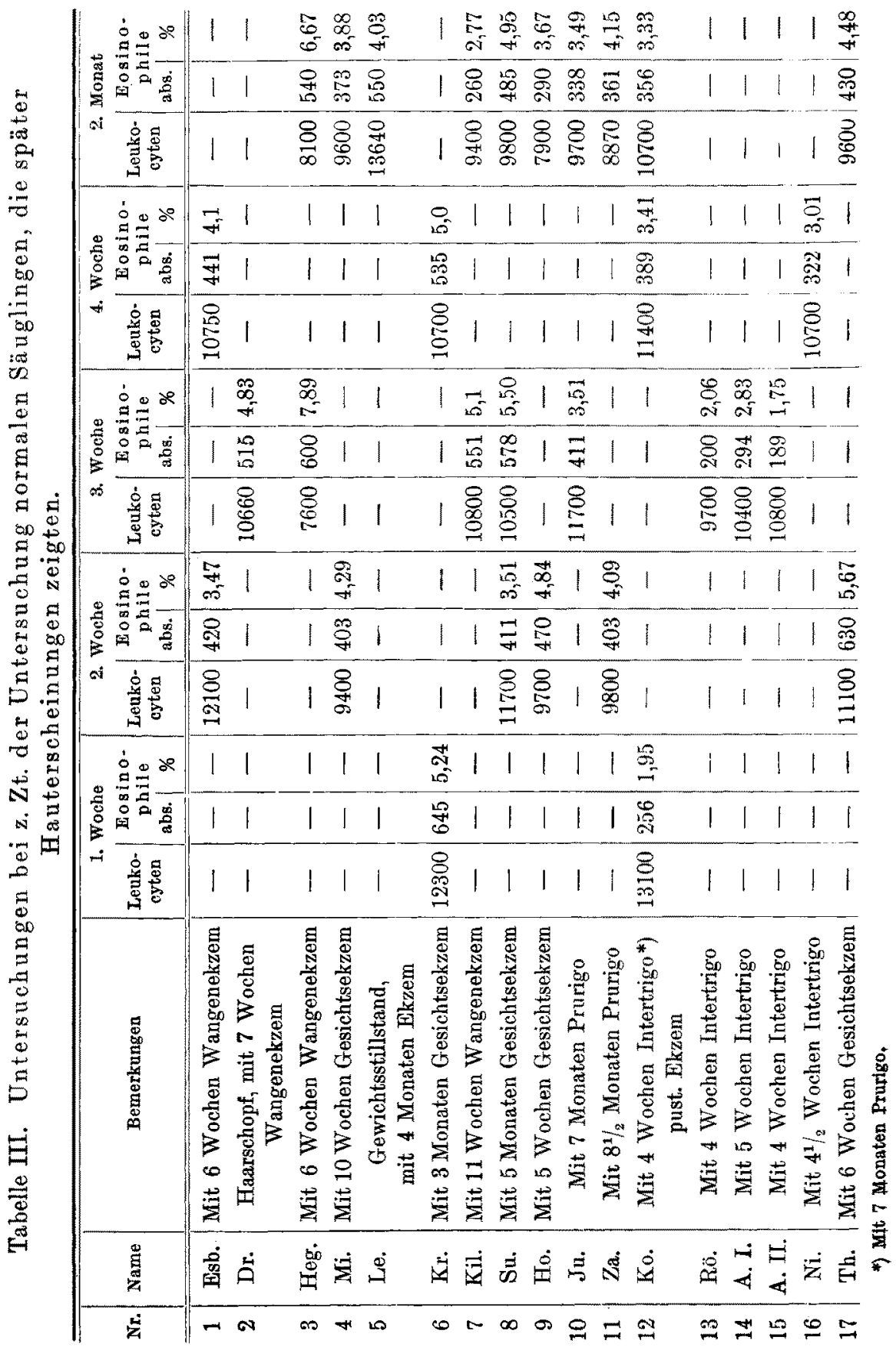


3. Kind F. A. Angeborem Gallengansverschluß.

5. XII. 1912. 15900 Leukoeyten, davon $37,81 \%$ mononucleäre und $62,19 \%$ polynucleäre, 1710000 rote Blutkörperchen, keine eosinophilen Zellen.

4. Kind S. Anämie, Lues, Otitis media.

17100 Leukocyten, 22 Eosinophile $=0,13 \%$.

5. Kind F. J. Lues, Anämie, Cystitis.

28. X. 1912. 10 Uhr a. m. 14600 Levkocyten, 199 Eosino phile $=1,37 \%$.

28. X. 1912. 2 Stunden nach Injektion von $10 \mathrm{ccm}$ Menschenserum, 15400 Leukocyten, 39 Eosinophile $=0,27 \%$.

6. Kind P. Anämie, Rachitis.

22. X. 1912. 11600 Leukocyten, 80 Eosinophile $=0,7 \%$. 2 Stunden nach Seruminjektion.

7. Kind O. Anämie, Ernährungsstörung, Rachitis.

28. X. 1912. 14600 Leukocyten, 200 Eosinophile $=1,37 \%$.

29. X. 2 Stunden nach Seruminjektion: 15200 Leukocyten, 111 Eosinophile $=0,73 \%$.

2. XI. 16250 Leukocyten, 166 Eosinophile $=1,0 \% \mathrm{ca}$.

8. Kind N. Anämie, Rachitis, Pyelitis.

2. XIr. 13900 Leukoeyten, 240 Eosinophile $=1,73 \%$.

3. XII. 5 Stunden nach Seruminjektion, 21800 Leukocyten, 275 Eosinophile $=1,26 \%$.

9. Kind B. R. Sehwere Anämie und Rachitis.

8. X. 7890 Leukocyten, 52 Eosinophile $=0,66 \%$.

9. X. 2 Stunden nach Seruminjektion, 9844 Leukocyten, 594 Eosinophile $=6,03 \%$.

10. X. 9267 Leukocyten, 438 Eosinophile $=4,72 \%$.

29. X. 9650 Leukocyten, 490 Eosinophile $=5,08 \%$.

30. X. 2 Stunden nach Seruminjektion, 9900 Leakocyten, 360 Eosinophile $=3,64 \%$.

30. XII. Schwere Pneumonie, großer Milztumor, 24600 Leukocyten, 161 Eosinophile $=0,65^{\circ}$.

1. I. 1913. Exitus.

10. Kind B. O. Mit 3 Wochen Hämatom der Wange, zur Zeit der ersten Cntersuchung barlowartige Erscheinungen, Hämaturie usw. Temperatur $39,3^{\circ}$.

24. IX. 59700 Leukocyten, 60 Eosinophile $=0,1 \%$.

25. IX. 22500 Leukocyten, 52 Eosinophile $=0,23 \%$.

26. IX. Temperatur $38,9,21250$ Leukocyten, 42 Eosinophile $=0,2^{\circ} \circ$.

3. X. 1912. Keine neuen Blutungen, Temperatur normal. 10700 Leukocyten, 568 Eosinophile $=5,48 \%$.

14. XI. Neuerdings Hämatom der Wange. 24000 Leukocyten, 190 Eosino. phile $=0,79 \%$.

25. XI. 11700 Leukocyten, 527 Eosinophile $=4,5 \%$. Keine neuen Blutungen.

6. II. 1913. Kind hat in der Zwischenzeit keine neuen Blutungen gehabt. 10200 Leukocyten, 506 Eosinophile $=4,96 \%$. 
440 H. Putzig: Vorkommen und klinische Bedeutung der eosinophilen Zellen

11. Kind Ra. Im Hause geboren.

1. Untersuchung 5 Wochen alt. 9200 Leukocyten, 218 E $o$ sinophile $=2,37 \%$.

2. Untersuchung 4 Monate alt. Blaß, sonst ohne Beschwerden. 8300 Leukocyten, 274 Eosinophile $=3,30 \%$.

3. Untersuchung 6 Monate alt. Drüseneiterung am Hals. 16300 Leukocyten, 117 Eosinophile $=0,72 \%$.

12. Kind He. Im Hause geboren.

1. Untersuchung 10 Wochen alt. Blaß, obstipiert. Brust. 8500 Leukocyten, 212 Eosinophile $=2,49 \%$.

2. Untersuchung 4 Monate alt. Cystitis $38,3^{\circ}$. Brust. 7800 Leukocyten, 100 Eosinophile $=1,28 \%$.

3. Untersuchung 5 Monate alt. 1/2 Milch-Schleim. 10600 Leukocyten, 344 Eosinophile $=3,25 \%$.

4. Untersuchung 6 Monate alt. $1 / 2$ Milch-Schleim. Intertrigo. 7100 Leukocyten, 118 Eosinophile $=1,66^{\circ}{ }_{\mathrm{o}}$.

5. Untersuchung 8 Monate alt, mit Tuberkulose, ron außen wieder aufgenommen. 9100 Leukocyten, 215 Eosinophile $=2,36^{\circ}$.

Im ganzen haben also die Untersuchungen an diesen kranken Säuglingen nichts Besonderes ergeben. Sie bestätigen im allgemeinen die Resultate früherer Untersucher. Infektionskrankheiten, bzw. fieberhafte Affektionen, wie Abscesse, Cystitis usw., schwere Ernährungsstörungen, z. T. auch Anämie und Lues und Tuberkulose zeigen niedrige Werte, desgleichen die Hämophilie zur Zeit des Auftretens der Blutungen, während bei der Resorption der Blutergüsse sich relativ hohe Werte finden. Zweifelhaft sind die Ergebnisse nach Seruminjektionen, z. T. leichte Verminderung, z. T. Erhöhung.

Wir kommen nunmehr zu der vierten und interessantesten Gruppe unserer Untersuchungen, nämlich zu den Säuglingen mit Erscheinungen von exsudativer Diathese. Wenn man die Resultate der letzten Gruppe, deren Einzelergebnisse in Tabelle IV zusammengestellt sind, kurz resümiert, so ergibt sich folgendes:

Die Kinder mit Gneis, Milchschorf, trockenem, aus makulo-papulösen Efflorescenzen konfluierendem Körperekzem, d. h. Affektionen, bei denen die Exsudation, die Ausschwitzung im Vordergrunde steht, deren typische, oft symmetrische Lokalisation an das Blut- und Lymphgefäßsystem gebunden zu sein scheint, zeigen deutliche Eosinophilie. Diese Ekzeme entstehen unabhängig von äußerer Reizwirkung, können allerdings sekundär infiziert werden, wobei die Eosinophilenwerte zwar oft abnehmen, aber gegen die Norm erhöht bleiben. 
Tabelle IV. Säuglinge mit, ,exsudativer Diathese“.

\begin{tabular}{|c|c|c|c|c|c|}
\hline $\mathrm{Nr}$. & Name & Bemerkungen über den Verlauf & Leukocyten & $\begin{array}{c}\text { Eosinophile } \\
\text { absolut }\end{array}$ & $\begin{array}{l}\text { Eosinophile } \\
\text { relativ \% }\end{array}$ \\
\hline 1 & Co. & $\begin{array}{c}\text { Ekzema universale vom } \\
\text { Intertrigo aus }\end{array}$ & 12900 & 90 & 0,7 \\
\hline \multirow{6}{*}{2} & \multirow{6}{*}{ Ta. } & Ekzem abgeheilt & 9100 & 70 & 0,77 \\
\hline & & Desgl., 3 Monate später & 7040 & 81 & 1,15 \\
\hline & & $\begin{array}{c}\text { Schweres intertriginöses } \\
\text { Ekzem }\end{array}$ & 11700 & 120 & 1,02 \\
\hline & & $\begin{array}{c}\text { Gewichtsstillstand, Ekzem } \\
\text { unverïndert }\end{array}$ & 12300 & 105 & 0,85 \\
\hline & & $\begin{array}{c}\text { Bei Zunahme Abheilung } \\
\text { des Ekzems }\end{array}$ & 10300 & 172 & 1,67 \\
\hline & & Ekzem abgeheilt, Blasse & 10400 & 165 & 1,59 \\
\hline \multirow[t]{2}{*}{3} & \multirow[t]{2}{*}{ Re. } & $\begin{array}{c}\text { Schweres intertriginöses } \\
\text { Ekzem }\end{array}$ & 16690 & 0 & 0 \\
\hline & & $\begin{array}{c}\text { Ekzen gebessert, geringe } \\
\text { Dyspepsie }\end{array}$ & 12800 & 85 & 0,67 \\
\hline 4 & Sa. & $\begin{array}{c}\text { Schon mehrach Intertrigo, } \\
\text { jetzt wieder }\end{array}$ & 11700 & 89 & 0,76 \\
\hline \multirow{3}{*}{5} & & Intertrigo abgeheilt & 9700 & 135 & 1,39 \\
\hline & $\begin{array}{l}\text { Ko. } \\
\text { S. Tab.III,Nr. } 12\end{array}$ & Geringer Intertrigo & 11400 & 389 & 3,41 影最 \\
\hline & & Intertrigo abgeheilt & 10700 & 356 & $3,33]=$ \\
\hline 6 & $\begin{array}{c}\text { Rö. } \\
\text { S. Tab.III, Nr.13 }\end{array}$ & Leichter Intertrigo & 10100 & 185 & 1,83 \\
\hline 7 & $\begin{array}{c}\text { A. I. } \\
\text { S.Tab.III, Nr. } 14\end{array}$ & Stark nässender Intertrigo & 11800 & 225 & 1,91 \\
\hline 8 & $\begin{array}{c}\text { A. II. } \\
\text { S. Tab.III, Nr.15 }\end{array}$ & Geringer Intertrigo & 10700 & 156 & 1,46 \\
\hline 9 & Me. & $\begin{array}{c}\text { Leichtes Wangenekzem, } \\
\text { Intertrigo, Dyspepsie bei } \\
\text { Brustmilch }\end{array}$ & 12400 & 781 & 6,30 \\
\hline 10 & Be. & $\begin{array}{c}\text { Fruher Furunkulose, jetzt } \\
\text { Gesichtsekzem, fleckiges } \\
\text { Körperekzem }\end{array}$ & 18590 & 4094 & 22,02 \\
\hline & & Ekzem gebessert & 14500 & 2400 & 16,55 \\
\hline 11 & We. & $\begin{array}{c}\text { Schweres impetiginöses } \\
\text { symmetrisches Wangen- } \\
\text { ekzem }\end{array}$ & 17800 & 518 & 2,9 \\
\hline & & $\begin{array}{l}\text { Ekzem, trockener, } \\
\text { schuppend }\end{array}$ & 12100 & 740 & 6,12 \\
\hline 12 & $\begin{array}{c}\text { Esb. } \\
\text { S. Tab. III, Nr. } 1\end{array}$ & $\begin{array}{c}\text { Geringes symmetrisches } \\
\text { Wangenekzem }\end{array}$ & 9500 & 645 & 6,79 \\
\hline 13 & $\begin{array}{c}\text { Dreg. } \\
\text { S. Tab. III, Nr. } 2\end{array}$ & $\begin{array}{c}\text { Typisches Wangenekzem, } \\
\text { Haarschopf }\end{array}$ & 9800 & 610 & 6,22 \\
\hline
\end{tabular}


442 H. Putzig: Vorkommen und klinische Bedeutung der eosinophilen Zellen

Tabelle IV. (For tsetzung.)

\begin{tabular}{|c|c|c|c|c|c|}
\hline Nr. & Name & Bemerkungen über den Verlauf & Leukocyten & $\begin{array}{c}\text { Eosinophile } \\
\text { absolut }\end{array}$ & $\begin{array}{l}\text { Eosinophile } \\
\text { relativ \% }\end{array}$ \\
\hline \multirow[t]{2}{*}{14} & \multirow[t]{2}{*}{ Je. } & $\begin{array}{c}\text { Pustulöses Wangenekzem, } \\
\text { Dyspepsie }\end{array}$ & 8260 & 256 & 3,12 \\
\hline & & $\begin{array}{c}\text { Dyspepsie geheilt, } \\
\text { trockenes Gesichtsekzem }\end{array}$ & 8400 & 486 & 5,78 \\
\hline 15 & $\begin{array}{c}\text { Le. } \\
\text { S. Tab. III, Nr. } 5\end{array}$ & $\begin{array}{c}\text { Geringes Wangenekzem, } \\
\text { etwas Intertrigo }\end{array}$ & 9300 & 412 & 4,43 \\
\hline 16 & Po. & $\begin{array}{c}\text { Gesichtsekzem, geringe } \\
\text { Enteritis }\end{array}$ & 8670 & 471 & 5,43 \\
\hline \multirow[t]{5}{*}{17} & \multirow[t]{5}{*}{ Jec. } & $\begin{array}{l}\text { Trockenes, symmetrisches } \\
\text { Gesichtsekzem, fleckiges, } \\
\text { schuppendes Kürperekzem }\end{array}$ & 16400 & 1040 & 6,34 \\
\hline & & $\begin{array}{c}\text { Gesichtsekzem stärker, } \\
\text { Juckreiz, Körperekzen } \\
\text { unverändert }\end{array}$ & 15700 & 2150 & 13,69 \\
\hline & & Ekzem besser & 8600 & 890 & 10,34 \\
\hline & & $\begin{array}{c}\text { Ekzem fast verschwunden, } \\
\text { leichte Grippe }\end{array}$ & 10600 & 400 & 3,77 \\
\hline & & $\begin{array}{l}\text { Ekzem geheilt, keine } \\
\text { Grippe }\end{array}$ & 8800 & 645 & 7,33 \\
\hline \multirow[t]{2}{*}{18} & \multirow[t]{2}{*}{ Ar. } & $\begin{array}{c}\text { Typisches Wangenekzem, } \\
\text { diffuses fleckiges Körper- } \\
\text { ekzem }\end{array}$ & 15670 & 5220 & 33,3 \\
\hline & & $\begin{array}{c}\text { Starke Grippe, Furun- } \\
\text { kulose, Ekzem viel besser }\end{array}$ & 34700 & 430 & 1,24 \\
\hline \multirow[t]{2}{*}{19} & \multirow[t]{2}{*}{ Bc. } & $\begin{array}{l}\text { Typisches Gesichtsekzem } \\
\text { u. Gneis, Mutter exsudativ }\end{array}$ & 12700 & 2300 & 18,11 \\
\hline & & $\begin{array}{l}\text { Gesichtsekzem geringer, } \\
\text { Ekzerna varioliforme }\end{array}$ & 10900 & 516 & 4,73 \\
\hline \multirow[t]{5}{*}{20} & \multirow[t]{5}{*}{ Zo. } & $\begin{array}{c}\text { Starker Intertrigo bis zur } \\
\text { Nabelhöhe, typisches Ge- } \\
\text { sichtsekzem }\end{array}$ & 14300 & 372 & 2,60 \\
\hline & & $\begin{array}{c}\text { Intertrigo heilt } a b \\
\text { Gesichtsekzem stärker }\end{array}$ & 12760 & 589 & 4,64 \\
\hline & & $\begin{array}{l}\text { Körper ohne Ekzem, nur } \\
\text { Gesichtsekzem }\end{array}$ & 10500 & 911 & 8,68 \\
\hline & & $\begin{array}{c}\text { Grippe, Gesichtsekzem } \\
\text { besser }\end{array}$ & 15300 & 178 & 1,14 \\
\hline & & $\begin{array}{l}\text { Grippe abgeheilt, Ekzem } \\
\text { verschwunden }\end{array}$ & 10100 & 620 & 6,14 \\
\hline 21 & Ey. & $\begin{array}{l}\text { Hirschsprung, seit einigen } \\
\text { Tagen Gneis + makulo- } \\
\text { krustöses Gesichtsekzem }\end{array}$ & 7100 & 89 & 1,25 \\
\hline
\end{tabular}


im Säuglingsalter, besonders bei der exsudativen Diathese.

Tabelle IV. (Fortsetzung.)

\begin{tabular}{|c|c|c|c|c|c|}
\hline Nr. & Name & Bemerkungen über den Verlauf & I.eukocyten & $\begin{array}{c}\text { Eosinophile } \\
\text { absolut }\end{array}$ & $\begin{array}{l}\text { Eosinophile } \\
\text { relativ \% }\end{array}$ \\
\hline \multirow[t]{5}{*}{21} & \multirow[t]{5}{*}{$\begin{array}{c}\text { Ey. } \\
\text { (Fortsetzung) }\end{array}$} & $\begin{array}{c}\text { Gesichtsekzem impetiginös. } \\
\text { Temp. } 38,2\end{array}$ & 10200 & 265 & 2,52 \\
\hline & & $\begin{array}{c}\text { Ekzem besser. Erysipel des } \\
\text { rechten Armes. Temp. } \\
\text { septisch }\end{array}$ & 31700 & 106 & 0,33 \\
\hline & & $\begin{array}{c}\text { Erysipel ahgeheilt. Impeti- } \\
\text { ginöses Ekzem des Ge- } \\
\text { sichtes }\end{array}$ & 13500 & 1210 & 8,96 \\
\hline & & $\begin{array}{c}\text { Ekzem auf Pellidol ge- } \\
\text { bessert }\end{array}$ & 9000 & 1050 & 11,67 \\
\hline & & $\begin{array}{l}\text { Ekzem otwas stärker, } \\
\text { nur wenig impetiginos }\end{array}$ & 16200 & 1860 & 11,47 \\
\hline 22 & Wi. & $\begin{array}{l}\text { Nervöses Erbrechen. } \\
\text { Geringes Wangenekzem }\end{array}$ & 8800 & 528 & 6,0 \\
\hline 23 & Schr. & $\begin{array}{c}\text { Nervöses Erbrechen. } \\
\text { Typisches Gesichtsekzem }\end{array}$ & 8900 & 556 & 6,25 \\
\hline \multirow[t]{2}{*}{24} & \multirow[t]{2}{*}{ Leu. } & $\begin{array}{l}\text { Wangenekzem. Interm } \\
\text { trigo in Heilung }\end{array}$ & 10100 & 556 & 5,50 \\
\hline & & Prurigo. Gneis & 9600 & 618 & 6,44 \\
\hline \multirow[t]{7}{*}{25} & \multirow[t]{7}{*}{ Er } & $\begin{array}{c}\text { Primäres typisches Ge- } \\
\text { sichtzekzem. Makuloses } \\
\text { Ekzem an Körper }\end{array}$ & 18000 & 4950 & 27,5 \\
\hline & & $\begin{array}{l}\text { Gesichtsekzem impetiginös. } \\
\text { Körperekzem unverändert. }\end{array}$ & 16700 & 4370 & 26,17 \\
\hline & & $\begin{array}{c}2 \text { Monate spiter Gesichts. } \\
\text { ekzen: auf Pellidol etwas } \\
\text { brsser }\end{array}$ & 12300 & 1070 & 8,7 \\
\hline & & $\begin{array}{c}\text { Adrenalinbehandlung. } \\
\text { Das Körperekzem heilt ab. }\end{array}$ & 14200 & 860 & 6,06 \\
\hline & & \begin{tabular}{|} 
Adrenalintherapie Körper- \\
ekzem verschwunden, \\
Gesichtsekzem gebessert
\end{tabular} & 15100 & 750 & 4,97 \\
\hline & & $\begin{array}{c}\text { Starke Enteritis. Ekzem } \\
\text { verschwunden }\end{array}$ & 12700 & 218 & 1,72 \\
\hline & & $\begin{array}{l}\text { Enteritis abgeheilt. } \\
\text { Gesichtsekzem tritt auf }\end{array}$ & 11800 & 920 & 7,79 \\
\hline 26 & $\mathrm{Ku}$. & $\begin{array}{c}\text { Typisches Gesichtsekzem, } \\
\text { kein Körperekzem }\end{array}$ & 13500 & 700 & 5,19 \\
\hline \multirow[t]{2}{*}{27} & \multirow[t]{2}{*}{ Ot. } & $\begin{array}{c}\text { Gueis, geringes Gesichts- } \\
\text { ekzem }\end{array}$ & 12300 & 1220 & 9,92 \\
\hline & & $\begin{array}{c}\text { Grippe, Temp. 39,4 } \\
\text { Ekzem geringer }\end{array}$ & 15600 & 340 & 2,18 \\
\hline
\end{tabular}


444 H. Putrig: Vorkommen und klinische Bedeutung der eosinophilen Zellen

Tabelle IV. (Fortsetzung.)

\begin{tabular}{|c|c|c|c|c|c|}
\hline $\mathrm{N}_{\mathrm{r}}$ & Name & Bemerkungen uber den Verlauf & Leukocyten & $\begin{array}{c}\text { Eosinophile } \\
\text { absolut }\end{array}$ & $\begin{array}{c}\text { Eosinophile } \\
\text { relativ o: }\end{array}$ \\
\hline 27 & $\begin{array}{c}\text { Ot. } \\
\text { (Fortsetzung) }\end{array}$ & $\begin{array}{c}\text { Grippe abgeheilt. Ge- } \\
\text { sichtsekzem }\end{array}$ & 11300 & 970 & 8,58 \\
\hline 28 & $\begin{array}{l}\text { Th. } \\
\text { S.Tab.III,Nr. } 17\end{array}$ & Gneis, Gesichtsekzem & 8400 & 570 & 6,78 \\
\hline 29 & Ker. & $\begin{array}{l}\text { Starkes Gesichtsekzem. Ge- } \\
\text { ringer Intertrigo am Hals. }\end{array}$ & 8100 & 727 & 9,0 \\
\hline \multirow[t]{5}{*}{30} & \multirow[t]{5}{*}{$\mathrm{Kl}$. } & $\begin{array}{c}\text { Typisches Gesichtsekzem, } \\
\text { trockenes, fleckiges Körper- } \\
\text { ekzem }\end{array}$ & 9300 & 1100 & 11,83 \\
\hline & & $\begin{array}{c}\text { Besserung auf Adrenalin } \\
+ \text { Pellidol }\end{array}$ & 21700 & 720 & 3,32 \\
\hline & & $\begin{array}{l}\text { Verschlimmerung des } \\
\text { Gesichtsekzems }\end{array}$ & 14800 & 1750 & 11,82 \\
\hline & & Besserung des Ekzems & 12300 & 1310 & 10,65 \\
\hline & & Ekzem abgeheilt & 10400 & 860 & 8,27 \\
\hline \multirow[t]{4}{*}{31} & \multirow[t]{4}{*}{ BI. } & $\begin{array}{l}\text { Impetiginöses Gesichts- } \\
\text { ekzem. Furunkulose }\end{array}$ & 12700 & 110 & 0,87 \\
\hline & & $\begin{array}{c}\text { Furunkel abgeheilt. Ge- } \\
\text { sichtsekzem }\end{array}$ & 9700 & 930 & 9,59 \\
\hline & & $\begin{array}{c}\text { Geringes Gesichtsekzem. } \\
\text { Prurigo }\end{array}$ & 8900 & 1050 & 11,79 \\
\hline & & Ekzem und Prurigo geheilt & 9100 & 840 & 9,23 \\
\hline 32 & Th. & $\begin{array}{c}\text { Prurigo, Lingua geogra- } \\
\text { phica, früher Gesichtsekzem }\end{array}$ & 7650 & 384 & 5,02 \\
\hline 33 & Rö. & $\begin{array}{c}\text { Multiple Drüsenschwel- } \\
\text { lungen. Lingua geographica }\end{array}$ & 5900 & 156 & 2,64 \\
\hline & & id. & 6300 & 218 & 3,46 \\
\hline 34 & $\begin{array}{c}\text { Za. } \\
\text { S.Tab. III, Nr.11 }\end{array}$ & Kräftiges Kind. Prurigo & 8200 & 485 & 5,91 \\
\hline 35 & $\stackrel{\text { Ju. }}{\text { S.Tab. IIr, Nr, } 10}$ & Prurigo & 7400 & 375 & 5,09 \\
\hline 36 & Fr. & $\begin{array}{l}\text { Früher Gesichtsekzem, } \\
\text { jetzt Bronchitis } 39,3^{\circ} \\
\text { Bronchitis geheilt }\end{array}$ & $\begin{array}{l}27900 \\
11300\end{array}$ & $\begin{array}{r}44 \\
622\end{array}$ & $\begin{array}{l}0,16 \\
5,50\end{array}$ \\
\hline 37 & Erd. & $\begin{array}{l}\text { Geringer Gneis. Chro- } \\
\text { nische Bronchitis }\end{array}$ & 9920 & 328 & 3,3 \\
\hline
\end{tabular}

Im Gegensatz dazu haben Kinder mit ausgesprochen intertriginösem, nässendem Ekzem, bei denen die äußere Reizwirkung (schlechte Pflege, Durchfälle, Speien) eine 
auslösende Rolle spielt, keine Vermehrung der Eosinophilen, z. T. sogar Verminderung.

Interessant ist in dieser Hinsicht vor allem der Fall Z., Tab. IV Nr. 20, wo wir bei bestehendem Intertrigo niedrige Werte fanden, während bei Abheilen des Intertrigos und stärkerem Hervortreten des Gesichtsekzems eine deutliche Eosinophilie sich herausbildete.

Nach Abheilen des Ekzems bestand noch einige Zeit eine Vermehrung der eosinophilen Zellen fort.

Bei Prurigo, Lingua geographica, Urticaria fand ich nicht immer deutliche Verehrung der eosinophilen Zellen, allerdings Werte, die an der oberen Grenze des Normalen lagen.

Eine deutliche Verringerung der Eosinophilie, zum Teil unter normale Werte, konnte ich in allen Fällen feststellen, wo bei exsudativen Kindern interkurrente Infektionen, bzw. schwere Verdau ungsstörungen auftraten, wie dies z. B. auch bei Wurmkrankheiten mit Eosinophilie der Fall ist. (s. Stäubli, Gaisböck). In besonders typischer Weise zeigte sich der Einfluß der Infektion auf das Blutbild und Ekzem bei dem Fall A. (Tab. IV, Nr. 18), mit typischem Ekzem, besonders des Gesichts und des Kopfes, starker Eosinophilie, wo bei Auftreten einer schweren Sepsis, die zum Tode führte, eine starke Verminderung der Eosinophilen zugleich mit eine Besserung des Ekzems bemerkt wurde.

Ähnliches zeigte sich in dem Fall 0 , Tab. IV, Nr. 27, der bei interkurrenter Grippenaffektion Verminderung des Ekzems und der Eosinophilie zeigte.

Für die ganze Auffassung der Eosinophilie und ihrer Beziehung zur obwaltenden Diathese besonders wichtig erscheint uns der Fall Ey., Tab. IV, Nr. 21, bei dem, wie aus der Tabelle zu ersehen ist, zunächst geringes Ekzem bestand, das dann allmählich schlimmer wurde und das ganze Gesicht in typischer Weise einnahm. In dieser Zeit konnte durch wiederholte Untersuchungen niemals eine Vermehrung der eosinophilen Zellen, zum Teil sogar eine Verminderung festgestellt werden. Nach ungefähr dreiwöchiger Behandlung akquirierte das Kind ein Erysipel, während dessen Dauer sich weiterhin eine Verminderung der eosinophilen Zellen und zugleich eine Besserung des Gewichtsekzems zeigte. Nach der Abheilung des Erysipels erneutes Aufflammen des Ekzems, mit starker, monatelang beobachteter, konstanter Eosinophilie. 
Dies zeigt mit voller Deutlichkeit, daß nicht das Ekzem als solches die Eosinophilie bewirkt, sondern daß bei der Entstehung der Eosinophilie Vorgänge mitspielen, die wir vorläufig nicht übersehen können, die aber sicher nicht mit der Hauterscheinung gleichmäßig verlaufen.

\section{Eosinophilie und exsudative Diathese.}

Wir kommen damit zu der Frage nach der Bedeutung der Eosinophilie und zu ihren. Beziehungen zur exsudativen Diathese, sowie zu einigen, allerdings hauptsächlich theoretischen Auseinandersetzungen über die exsudative Diathese selbst. Die wissenschaftliche Streitfrage, um die es sich seit Jahren dabei handelt, ist die: Stellt die Eosinophilie ein Symptom der exsudativen Diathese dar, d. h. ist sie dem Ekzem gleichgeordnet oder ist sie abhängig von dem Ekzem, d.h. von der Hauterscheinung als solcher?

$\mathrm{Zu}$ der ersten Ansicht, daß die Eosinophilie ein Symptom der exsudativen Diathese sei, neigt vor allem Rosenstern, dem sich eine große Anzahl anderer Autoren, besonders auch die Verfasser der Lehrbücher für Kinderheilkunde, wie Heubner, Finkelstein u. a., anschließen. Seine Ansicht stützt sich vor allem auf die von ihm gefundenen Normalwerte, die mit den unsrigen nahezu übereinstimmen, weiterhin auf das Ergebnis, daß neben dem Ekzem vor allen Dingen das Asthma und andere Symptome der exsudativen Diathese, z. B. die Darmerscheinungen, Eosinophilie zeigen, und auf die Beobachtung, daß mit dem Abheilen des Ekzems nicht immer die Eosinophilie verschwindet, daß sie überhaupt nicht konform ist mit der Stärke der Hauterscheinungen.

Im Gegensatz zu Rosenstern steht besonders Aschenheim, dessen Ergebnisse sich aber, wie wir gleich hier vorausschicken müssen, auf ganz andere Normalwerte stützen, nämlich auf die von Benjamin gefundene Durchschnittszahl von $7,4 \%$, die nach unseren Untersuchungen sicher viel zu hoch liegt. Seine Ansicht, daß die Eosinophilie nur eine Folge der Hauterscheinungen sei, stützt sich weiter besonders auf die Feststellung, daß die Eosinophilie bei den anderen Erscheinungen der exsudativen Diathese gar nicht oder inkonstant vorhanden sei, daß weiterhin der familiäre Charakter fehle.

Wenn wir nun näher auf diese Frage eingehen, so ist zuzugeben, daß eine einwandfreie Lösung, wie Benfey hervorhebt, nur möglich 
wäre, wenn man bei einem von Geburt an beobachteten Kinde schon vor dem Auftreten der Hauterscheinungen eine Eosinophilie fände. Allerdings beweist das Fehlen der Eosinophilie bei der Geburt nichts gegen exsudative Diathese, da wir auch Ekzeme ohne Eosinophilie gefunden haben. Außerdem ist die Diathese ja keine Krankheit, sondern eine Disposition, die eines auslösenden Moments bedarf, um Erschęinungen zu machen.

Ich habe nun bei einer Reihe von Kindern, die später die oben erwähnten primär-exsudativen Erscheinungen zeigten, schon vor dem Auftreten der Hauterscheinungen zwar keine deutliche Eosinophilie, aber besonders in der 2 . bis 4 . Lebenswoche auffallend hohe, jedenfalls über die Norm hinausgehende Werte feststellen können. Bei Kindern, die später nur Intertrigo bekamen, fehlte diese Erhöhung.

Gegen die Argumentierung Aschenheims ist auch sonst verschiedenes einzuwenden.

1. Wenn auch die Eosinophilie bei floridem Ekzem häufiger ist als bei den übrigen Erscheinungen der exsudativen Diathese, so ist sie doch, wie andere Untersucher, z. B. Langstein, Noorden, Peter, Strü m pell usw., gefunden haben, auch bei den übrigen Erscheinungen dieser Diathese sehr häufig, und man könnte mit demselben Rechte also Asthma, eosinophile Darmkrisen, Prurigo und andere Erscheinungen der exsudativen Diathese nicht als gleichwertiges Symptom ansehen.

Wie Tandler sehr richtig bemerkt, betrifft eine konstitutionelle Eigenschaft sä mtliche Organe des betreffenden Individuums, wenn sie natürlich auch in den verschiedenen Organen verschieden stark in die Erscheinung tritt. Der Grad dieses Hervortretens gibt dann das hervorstehendste Symptom der betreffenden Anomalie, wie wir denn auch bei der exsudativen Diathese bald mehr Hauterscheinungen, bald mehr Erscheinungen von seiten der Schleimhäute, teilweise aber auch allgemeine Entwicklungsstörungen beobachten können.

2. Die Schlüsse, die Aschenheim zieht, gehen von zu hohen Normalzahlen a us - bis $9,3 \%$ - während nach unseren Untersuchungen Zahlen von 4,0\% und darüber, besonders bei den hohen Leukocyten-Werten, die Asche nheim fand, als über die Norm hinausgehend anzusehen sind. Damit würde ein Teil der Kinder, bei denen nach Aschenheims Ansicht die Eosinophilie fehlt, doch eine Vermehrung dieser Zellen über die Norm zeigen.

3. Das Verschwinden der easinophilen Zellen mit dem Abklingen des Ekzems ist nicht konstant. In vielen Fällen besteht die Eosinophilie 
weiter, nachdem das Ekzem schon abgeheilt ist, wenn auch die Werte der Eosinophilen immerhin abnehmen. Sie kehren erst eine geraume Zeit nach Verschwinden der Hauterseheinung zur Norm zurück. In einigen Fällen bleibt dauernd eine Erhöhung der Eosinophilenwerte erhalten.

Weiter fanden wir, daß die Eosinophilie bei Affektionen, die beim normalen Kinde eine Verminderung der Eosinophilie bewirken, auch beim esxudativen Kinde schon vor Abheilen des Ekzems abnahm (Adrenalininjektion, akute Infektion). Für die Unabhängigkeit der eosinophilen Zellen vom Ekzem als Hauterscheinung spricht weiterhin, daß die eosinophilen Zellen beim Ekzem lange Zeit fehlen können (s. Fall Ey.), um dann plötzlich bei einem neuen Ekzemschub aufzutreten. Sie sind also nicht von der Hauterscheinung und ihrer Intensität abhängig, sondern für ihre Vermehrung kommen eben noch unbekannte, vielleicht sensibilisierende Vorgänge in Betracht. Auch der Umstand, daß familiäre Eosinophilie, wie Aschenheim meint, nicht nachzuweisen ist ${ }^{1}$ ), wäre in diesem Sinne erklärlich dadurch, daß eben in diesen Fällen eine vorherige Sensibilitation fehlt. Außerdem sind von verschiedenen Autoren (Gangain und Klinkert) typische Fälle von familiärer Eosinophilie gefunden worden.

Im ganzen kommen wir auf Grund unserer Untersuchungen und der Betrachtung der vorliegenden Literatur zu dem Schluß, daß die Eosinophilie nicht vom Ekzem abhängig, sondern ein Symptom der exsudativen Diathese ist.

Ich möchte sogar noch weitergehen. Die Untersuchungen haben ergeben, daß die primär-exsudativen Erscheinungen, wie Milchschorf, Gneis, das aus makulo-papulösen Efflorescenzen konfluierende Körperekzem Eosinophilie zeigen, während beim nässenden Intertrigo diese fehlt. Sie haben weiter ergeben, daß nur die Kinder, welche später jene primär-exsudativen Symptome darboten, schon in der 2.-4. Woche erhöhte Eosinophilenwerte zeigten.

Auch klinisch verhält sich der Intertrigo, wie bekannt, anders. Er entsteht bei Affektionen (z. B. akuten Ernährungsstörungen, parenteralen

1) Der Fall von Dr. E. A. ist übrigens keineswegs beweisend. Er wäre höchstens im Sinne der konstitutionellen Bedeutung der Eosinophilie zu verwerten; denn es besteht in diesem Falle gleichzeitig ein hoher Grad von Mononucleose, der entschieden für eine Konstitutionsanomalie im Sinne einer Störung der inneren Sekretion spricht, und der sich auch in einem anderen, von Aschenheim angeführten Falle von Eosinophilie beim Gesunden findet. 
Infekten) zu einer Zeit, wo gerade die primär-exsudativen Erscheinungen verschwinden, er heilt ab, z. B. in der Rekonvaleszenz von Ernährungsstörungen, zu einer Zeit, wo bekanntlich Gesichtsekzem usw. aufflammen. Seine Lokalisation fällt im Gegensatz zu den exsudativen Erscheinungen mit der Stelle des äußeren Reizes zusammen. Deshalb erscheint eine prinzipielle Trennung der oben zusammengestellten primär-exsudativen Erscheinungen und des Intertrigo notwendig. $\mathrm{Nur}$ jene Exsudationen sind ein Symptom der exsudativen Diathese, nicht der Intertrigo. Dieser erscheint vielmehr als eine durch äußere Reize (Bakterien usw.) auf empfindlicher, bzw. in ihrer Immunität geschädigter Haut hervorgerufene Entzündung, als Dermatitis. Erkann naturlich mit der exsudativen Diathese zugleich vorkommen, ist aber kein obligatorisches primäres Symptom. Für diese Ansicht sprechen auch die Befunde von Mautner, der bei primärem trockenem Ekzem keine besondere cutane Empfindlichkeit gegen Bakterienproteine feststellen konnte, während die Haut bei einem Kinde mit Intertrigo stark reagierte. Eine ähnliche Unterscheidung zwischen konstitutionellem und sekundärem Ekzem führt Bruck für die Erwachsenenekzeme durch. Auch Peter äußert sich in diesem Sinne auf Grund von Befunden, daß eine Vermehrung der eosinophilen Zellen sich niemals bei artifiziellen Ekzemen, sondern nur bei symptomatischen Formen finde, deren Ursache in einer nicht nachzuweisenden Schädigung des Organismus zu suchen sei.

Was für hypothetische Ausblicke gestattet uns dieses Vorkommen der Eosinophilie bei der exsudativen Diathese?

Die Eosinophile kommt, um kurz die Ergebnisse früherer Untersucher zusammenzufassen, besonders vor bei Wurmkrankheiten, bei Asthma, verschiedenen Hautaffektionen, bei der Resorption von Blutergüssen usw. In neuester Zeit ist besonders durch die Untersuchungen von Schwenker und Schlecht auf das lokale und allgemeine Vorkommen dieser Zellen bei der Anaph yla xie aufmerksam gemacht worden. Die Injektion von artfremdem EiweiB, bzw. von EiweiBabbau-Produkten bewirkt neben den typisch anaphylaktischen Erscheinungen eine ausgesprochene Eosinophilie. Auch für die parasitären. Erscheinungen ist die Wirksamkeit von heterologen, bzw. durch die Parasiten heterolog gemachtem Eiweiß (Wolf-Eis ner) wahrscheinlich geworden durch Versuche, die ergeben haben, daß proteinfreies Askaridenextrakt keine Eosinophilie hervorruft. 
Wenn man sich die Symptome der Anaphylaxie ins Gedächtnis ruft, die symmetrisch lokalisierten Hauterscheinungen, die Lungenerscheinungen, die Darmerscheinungen mit Exsudation von eosinophilem Schleim, den Herzbefund im anaphylaktischen Shock und zuletzt die Eosinophilie und eine Parallele zieht zur exsudativen Diathese, so fällt eine bedeutende Übereinstimmung auf. Auch hier sehen wir die typische Lokalisation der Hauterscheinungen mit Freilassen des Mundes (wie übrigens auch beim Scharlach, dem einzigen akuten Exanthem mit Eosinophilie), die Lungenerscheinungen mit Lungenlähmung, den eosinophilen Darmkatarrh, die nervösen Reizsymptome und schließlich den Herzstillstand im Ekzemtod.

Diese Gegenüberstellung legt den. Schluß nahe, daß wir es bei der exsudativen Diathese, bzw. bei den oben erwähnten Symptomen dieser Diathese mit anaphylaxieähnlichen Erscheinungen zu tun haben. Wodurch diese ausgelöst sind, ob durch Störung des parenteralen Eiweißbaues, ob durch UUbergang von blut- bzw. organfremdem Eiweiß in das Blut, läßt sich jetzt noch nicht sagen. Die französischen Autoren (Galup u. a.), die in ähnlicher Weise Anaphylaxie und Lymphatismus bzw. Arthritismus zu identifizieren suchen, nehmen eine Störung der Schutzvorrichtungen des Organismus (Darm, Leber, Drüsen mit innerer Sekretion) als Ursache an.

Betrachten wir die exsudative Diathese nun unter dem Gesichtspunkt der Anaphylaxie, so finden sich weitere Ubereinstimmungen. Als eines der Hauptsymptome der Anaphylaxie gilt der starke primäre Juckreiz, der ja auch bei exsudativen Kindern oft schon vor Auftreten manifester Symptome, jedenfalls aber bei den Hauterscheinungen stark zutage tritt. Abgesehen von diesen nervösen Erscheinungen, steht bei der Anaphylaxie wie bei der exsudativen Diathese ein Utberwiegen des Vaguston us im Vordergrunde. Daß allerdings der Vagus nicht primär der Anlaß zu den Erscheinungen ist, ergibt sich daraus, daß es nicht gelungen ist, durch Vagusreizung ein Symptom wie Asthma, Eosinophilie usw. hervorzurufen, höchstens bestehende Erscheinungen zu beeinflussen. Wir müssen also annehmen, daß primär eine andere Störung im Organismus vorhanden ist. Auch hier finden sich Ähnlichkeiten zwischen Anaphylaxie und exsudativer Diathese.

Calvary hat nachgewiesen, daß die anaphylaktogenen Stoffe eine Vermehrung der Lymphmenge bewirken, und auch für die exsudative Diathese ist eine Vermehrung der Gewebsflüssigkeit und des Wassergehaltes des Blutes wiederholt (von Lust, zuletzt von Lederer) hervor- 
gehoben worden. Auch umgekehrt ist bekannt, daß Hunger, bzw. Wasserentziehung z. B. bei akuten Ernährungsstörungen die exsudativen Erscheinungen zum Schwinden bringt, ebenso wie es möglich ist, durch Hunger die Anaphylaxie zu vermeiden. Anzunehmen ist, daf auch der mit dem Wassergehalt eng verbundene Mineralstoffwechsel beteiligt ist. Jedenfalls ist es durch Salzinjektionen gelungen, die Anaphylaxie zu vermeiden (Langer) und die Eosinophilie zu vermindern (Rosenstern). Auch ist der Einfluß von Störungen des Mineralstoffwechsels auf die Erregbarkeit des autonomen Systems bekannt. Es sei hier im Zusammenhang erwähnt, daß schon Ehrlich nachgewiesen hat, daß die eosinophilen Zellen gequollen, $d . h$. wasserreicher sind, als die neutrophilen. Limbeck macht darauf aufmerksam, daß die Eiweißstoffe der eosinophilen Granula möglicherweise solche sind, die zu ihrer Lösung einer bestimmten Salzkonzentration bedürfen, also bei Wasseraufnahme und Quellung ausfallen. Dies nur nebenbei. Vielleicht ist auf diese Weise die erhöhte Zahl von Eosinophilen bei später exsudativen Kindern gerade in der zweiten bis vierten Woche zu erklären, wo die Gewichtszunahme nach der physiologischen Abnahme eintritt.

Im ganzen lassen die Übereinstimmungen zwischen exsudativer Diathese und Anaphylaxie die Auffassung der oben zusammengestellten primär-exsudativen Erscheinungen als Symptome einer protrahierten Anaphylaxie wohl berechtigt erscheinen.

Wir kommen endlich zu der Frage, welche therapeutischen Schlüsse uns diese Betrachtungen erlauben. Sie zeigen uns, daß man bei der Behandlung von Intertrigo einserseits und den exsudativen Erscheinungen andererseits ganz verschiedene Punkte berücksichtigen muß. Beim Intertrigo wird es sich darum handeln, die Immunität durch geeignete Ernährung (z. B. Frauenmilch) und ev. sogar reichliche Nahrungszufuhr zu heben und weiter die Hautempfindlichkeit zu verringern, vielleicht durch Calciumsalze, Seruminjektionen (s. die Untersuchungen von Luithlen). Daneben wird in diesen Fällen eine lokale Therapie, die die Reizwirkung ausschaltet, von Nutzen sein.

Bei den exsudativen Erscheinungen hingegen kommt diese lokale Therapie nur für sekundär infizierte Ekzeme in Frage. Eine kausale Therapie wird aber nur möglich sein, wenn es gelingt, die inneren Stoffwechselvorgänge umzustimmen. Wie weit dies möglich sein wird, ob durch eiweißarme Nahrung, wie sie Salomon für die Urticaria empfiehlt, ob durch knappe Ernährung, ob durch Wasserentziehung, durch Änderung des Mineralstoff wechsels, ist noch nicht zu entscheiden. Hier 
sei nur erwähnt, daß Kalksalze jedenfalls günstig wirken, und zwar in mehrfacher Beziehung, sie setzen die Hautempfindlichkeit herab, sie erhöhen die Vasokonstriktion und verhindern so die Exsudation und sie vermindern den Tonus des vegetativen Nervensystems, dessen Rolle oben erörtert ist.

Zur Herabsetzung des Vagustonus hat vor kurzem Krasnogors ki Atropin bei exsudativer Diathese empfohlen. Die Untersuchungen sind nicht einwandfrei genug, um weitere Schlüsse zu erlauben. Jedenfalls dürfte sich an gewissem Material eine Nachprüfung empfehlen, da auch ich schon vor Krasnogorski bei Adrenalinanwendung teilweise gute Erfolge beobachtet habe. Hier ist vielleicht eine Möglichkeit ebenso wie bei der Anaphylaxie (s. Friedberger), therapeutische Erfolge zu erzielen.

\section{Zusammenfassung.}

Die Untersuchungen haben ergeben:

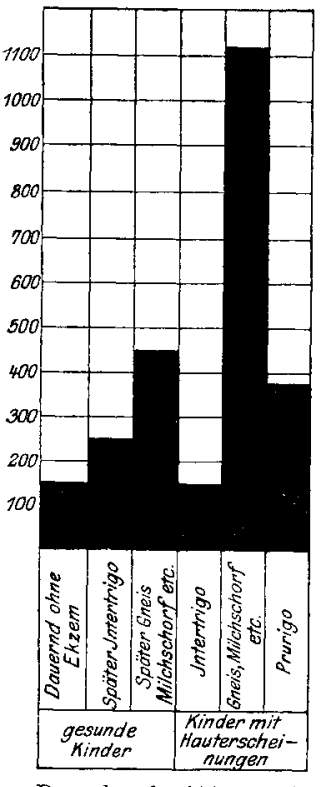

Durchschnittswerte der Eosinophilen procmm Blut

1. daß die Werte der eosinophilen Zellen beim gesunden normalen Säugling nicht höher liegen als beim gesunden Erwachsenen (2-4\%);

2. daß im allgemeinen die gesunden Säuglinge, bei denen später exsudative Erscheinungen sich gezeigt haben, schon frühzeitig und besonders in der zweiten bis vierten Woche höhere Maximal-und Durchschnittswerte aufweisen als gleichaltrige, vollkommen gesunde Säuglinge.

3. Die Untersuchungen an kranken, nicht exsudativen Säuglingen haben eine Verminderung bei akuten Infektionen und Ernährungsstörungen gezeigt, eine Vermehrung eigentlich nur in einem Falle von Hämophilie während der Resorption der Blutergüsse.

4. Von den untersuchten Säuglingen mit Hautveränderungen zeigten eine typische Eosinophilie diejenigen mit primär-exsudativen Erscheinungen (Milchschorf, Gneis), während die Fälle mit universellem in- 
fiziertem Ekzem, bzw. Intertrigo keine Eosinophilie a uf wiesen.

Die Eosinophilie verläuft nicht konform mit der Stärke der Hauterscheinungen und besteht meist noch eine Zeit nach Abheilen des Ekzems fort.

5. Die Eosinophilie ist also ein Symptom derexsudativen Diathese.

6. Die Untersuchungen zeigen von neuem, daß der Intertrigo eine Dermatitis ist, die auch bei exsudativer Diathese vorkommen kann, aber kein primäres obligatorisches Symptom ist.

7. Auf Grund hypothetischer Betrachtungen wird auf die große Ubereinstimmung zwischen Anaphylaxie und exsudativer Diathese hingewiesen, die vielleicht als eine Art protrahierter Anaphylaxie betrachtet werden kann.

\section{Literaturverzeichnis.}

Ahl und Schlittenhelm, Zeitschr. f. d. ges. exper. Med. 1, S. 111. 1913. Arneth, Deutsches Archiv f. klin. Med. 108, S. 323. 1912.

Arnold, Zentralbl. f. allg. Pathol. u. pathol. Anatomie 24, S. 673. 1913. Aschenheim, Deutsches Archiv f. klin. Med. 105, S. 470. 1912.

-, Monatsschr. f. Kinderheilk. 11, S. 269.1912.

- und Tomono, Monatsschr. f. Kinderheilk. 10, S. 340. 1912.

Audrain, J., Progr. méd. 41, S, 121. 1913.

Bahrdt und Benfey, Zeitschr. f. Kinderheilk. Orig. y, S. 481.1913.

Benfey, Monatsschr. f. Kinderheilk. 11, 421. 1913.

Benjamin, XXV. Versammlung d. Ges. f. Kinderheilk. S. 286. 1908.

Bettmann, Volkmanns Sammlung klin. Vortr. Nr. 266. 1900.

Biffi et Galie, Journ. de Physiol. et de Pathol. génér. 9, S. 721. - 1907.

Besnier, Ann. de Dermat. et de Syphil. 1891. S. 634.

Borchardt, Deutsches Archiv f. klin. Med. 106, S. 182. 1902.

Bruck, Beiträge z. Klin. d. Infektionskrankh. u. z. Immunitätsforschung 1, S. 549.1913.

Busch, Zeitschr. f. experim. Pathol. u. Ther. 14, S. 335. 1913.

Büttner, Wiener klin. Wochenschr. 26, S. 125. 1913.

Calvary, Münch. med. Wochenschr. 58, S. 670. 1911.

Caro, Berliner klin. Wochenschr. 49, S. 1514. 1912.

Carstanjen, Jahrb. f. Kinderheilk. 52, S. 215.1900.

Csepai, Deutsches Archiv f. klin. Med. 111, S. 271.1913.

Czerny, Jahrb. f. Kinderheilk. 61, S. 199. 1905.

—, Monatsschr. f. Kinderheilk. 7, S. 1. 1908.

Czerny-Keller, Des Säuglings Ernährungsstörungen usw.

Dunger, Münch. med. Wochenschr. 5\%, S. 1942. 1910. 
454 H. Putzig: Vorkommen und klinische Bedeutung der eosinophilen Zellen

Ebell, Zit. nach ZentralbI. f. d. ges. inn. Med. 3, S. 118.

Eckert, Grundriß d. Kinderheilk. Berlin 1912.

Eggers, Zeitschr. f. Röntgenkunde 15, S. 1. 1913.

Ehrlich, Archiv f. Anat. u. Physiol. 1879.

-, Zeitschr. f. klin. Medizin 1. 1880.

-, Farbenanalytische Untersuchungen. Gesammelte Mitteil. 1891.

- Lazarus, Die Anämie in Nothnagels Handbuch.

Elder and Hutchinson, Edinburgh Med. Journ. 1895.

Elzholz, Wiener klin. Wochenschr. $\gamma$, S. 587. 1894.

Eppinger, Med. Klin. 6, S. 922. 1910.

- und HeB, Die Vagotonie.

Feer, Erg. d. inn. Med. u. Kinderheilk. 8, S. 365. 1912.

Finday, Lancet 1, S. 1164. 1909; ref. Jahrb. f. Kinderheilk. $\boldsymbol{7 1}$, S. 782.

Finkelstein, Lehrbuch d. Säuglingskrankh. 2, S. 546. 1912.

Fischer, Zieglers Beiträge 55, S. 1. 1913.

Fischl, Prager med. Wochenschr. 1\%, S. 122. 1892.

-, Zeitschr. f. Heilkunde 1892. S. 277.

Friedberger und Langer, Zeitschr. f. Immunitätsf. 15, S. 535.1912.

Gaisböck, Therap. Monatshefte 26, Heft 8. 1912.

Gala mbos, Fol. haemat. 13, S. 269.

Galup, Presse méd. 21, S. 313. 1913.

Gangain, Bull. de la soc. de méd. d'Angers 1909; ref. Archiv f. Kinderheilk. 56, S. 212.

Gelbart, Korrespondenzbl. f. Schweiz. Ärzte 42, Nr. 29. 1912.

Goldzieher, Frankfurter Zeitschr. f. Pathol. 10, S. 174. 1912.

Grawitz, Charité-Annalen 26. 1891.

Gundobin, Jahrb. f. Kinderheilk. 35, S. 187. 1893.

—, Die Besonderheiten des Kindesalters. Berlin 1912.

Head, Pedriatrics 1900. Heft 3. Ref. Arch. f. Kinderheilk. 35, S. 146.

Helmholz, Jahrb. f. Kinderheilk. 69, S. 153. 1909.

Herrick, Aroh. of internal. med. 11, S. 165. 1913.

Hertzell, Inaug.-Diss. Leipzig. 10. Dez. 1907.

Heubner, Lehrbuch d. Kinderkrankh. 2, S. 35. 1911.

Hirschfeld, Monatsschr. f. Kinderheilk. 10, S. 38. 1911.

Hock, R., Centralbl. f. Bakt. u. Parasitenk. 54, S. 247. 1910.

— und Schlesinger, Beiträge zur Kinderheilk. N. F. II. Deuticke. Leipzig 1892.

Huguenin, Schweizer Rundschau 12, S. 809. 1912.

Jacksch, Klin. Diagnostik. VI. Aufl. S. 37. 1907.

Japha, Erkrank. des Blutes in Pfaundler-Schloßmann. III. Aufl. Bd. 2.

一, Jahrb. f. Kinderheilk. 52. S. 242.1900.

一, Jahrb. f. Kinderheilk. 53, S. 179. 1901.

Karnitzki, Archiv f. Kinderheilk. 36, S. 42.1903.

Kassowitz, Beiträge z. Kinderheilk. N. F. II. Deuticke. Leipzig 1892.

Klein, Zentralbl. f. inn. Med. 20, S. 96. 1899.

Klinkert, Berliner klin. Wochenschr. 48, S. 938. 1911.

Krasnogorski, Monatsschr. f. Kinderheilk. 12, S. 129. 1913.

Langer, Münch. med. Wochenschr. 59, S. 2554. 1912. 
Langstein, Berliner klin. Wochenschr. 45, S. 1218. 1908.

- Münch. med. Wochenschr. 58, S. 623. 1911.

Lanine, Arch. de biolog. 2\%, S. 525. 1912.

Lenzmann, Med. Klin. 9, S. 587. 1913.

Leuret et Gauvenet, Compt. rend. de la Soc. de Biol. 71, S. 677. 1911.

Limbeck, Grundriß einer klinischen Pathol. des Blutes. II. Aufl. Jena 1896. Longa, La Pediatria 1894. S. 72; ref. Jahrb. f. Kinderheilk. 43, S. 283.

Loos, XI. Versammlung d. Ges. f. Kinderheilk. 1894.

-, Jahrb. f. Kinderheilk. 39, S. 331. 1895.

Low, Journ. of stat. med. 20, S. 413. 1912; ref. Zentralbl. f. d. ges, inn. Med. 3, Heft 2.

Löwit, Zieglers Beiträge 10, S. 213. 1891.

Luithlen, Wiener klin. Wochensehr. 26, S. 682, 1913.

-, Wiener med. Wochenschr. 1913. Heft 38. S. 2375.

Luzet, Arch. génér. de méd. 27, S. 579. 1891.

Mautner, Zeitschr. f. Kinderheilk. Orig. 8, S. 461. 1913.

Mendel, Berliner klin. Wochensohr. 33, S. 1000. 1896.

Mennacher, Gesellsch. f. Kinderheilk. 1909.

Meyer, K., Die klinische Bedeutung der Eosinophilie. Berlin 1905.

Mosse-Grünba a m, Jahrb. f. Kinderheilk. 58, S. 435. 1903.

Müller, Wiener klin. Wochensehr. 26, S. 1025. 1913.

- uñd Rieder, Deutsches Archiv f. klin. Med. 48, S. 96.

Naegeli, Blutkrankheiten und Blutdiagnostik. S. 199. Leipzig 1912.

Neusser, Wiener klin. Wochenschr. 5, S. 41. 1892.

d'Orlandi, Rev. des malad. de l'enfance 1\%. 1899.

Ostrowski, Fol. haemat. 13, S. 305. 1912.

Pappenheim, Erg. d. inn. Med. u. Kinderheilk. 8, S. 183.1912.

Peter, Dermatolog. Zeitschr. 4, S. 669.1897.

Petry, Münch. med. Wochenschr. 59, S. 1892. 1912.

-, Biochem. Zeitschr. 38, S. 92. 1912.

Pfa undler in Feer, Lehrbuch d. Kinderheilk. S. 181. 1911.

Rekzeh, Deutsches Archiv f. klin. Med. rr. 1904.

Rieder, Zit. nach Carstanjen. 1892.

Ritz, Zeitschr. f. Immunitätsf. 12, S. 644. 1912.

Rosenstern, Jahrb. f. Kinderheilk. 69, S. 631. 1909.

-, Monatsschr. f. Kinderheilk. 8, S. 9. 1910.

Sahli, Lehrbuch d. klin. Untersuchungsmethoden.

Scipiades, Archiv f. Gynäkol. 70. 1904.

Schlecht, Archiv f. experim. Pathol. u. Pharmakol. 67, S. 137. 1912.

-, Kongr. f. inn. Med. 1910; ref. Berl. klin. Wochenschr. 47, S. 993.

-, Münch. med. Wochenschr. 60, S. 800. 1913.

- und Schwenker, Deutsches Archiv f. klin. Med. 108, S. 405. 1912.

Scholz, Centralbl. f. Bakt. u. Parasitenk. Orig. 65, S. 189. 1912.

Schüffner, Münch. med. Wochenschr. 58, S. 1451. 1911.

Schwarz, Wiener klin. Wochenschr. 24, S. 258. 1911.

Sch warze, G., Inaug.-Diss. Berlin 10. Aug. 1880 in Ehrlichs Farbenanalyt. Untersuchungen. 
Schwenker und Schlecht, Zeitschr. f. klin. Med. 76 , S. 7 r.

Siess und Störk, Wiener med. Wochenschr. 63, S. 1123.1913.

Skorzewski und Wasserberg, Zeitschr. f. experim. Pathol. u. Ther. 10, S. 330. 1912.

Spuler und Schittenhelm, Deutsches Archiv f. klin. Med. 109, S. 1. 1912. Stäubli, Erg. d. inn. Med. u. Kinderheilk. 6, S. 192.1910.

Steiger und Strebel, Zentralbl. f. d. ges. inn. Med. 34, S. 1049. 1913.

Strisower, Wiener klin. Wochenschr. 26, S. 16. 1913.

Strshelbizki, Bioblioth. Wratscha 1897; zit. nach Gundobin.

Strü m pell, Med. Klin. 6, S. 889.1910.

Takas u, Archiv f. Kinderheilk. 39, S. 346. 1904.

Tandler, Zeitschr. f. angew. Anat. u. Konstitutionslehre 1, S. 11.1913.

Türk, Vorlesungen über klin. Hämatologie. II. Teil, I. Hälfte. S. 270. 191\%.

Weidenreich, Die Leukocyten und verwandte Zellformen. Wiesbaden 1911.

Weinberg et Séguin, Compt, rend. de la Soc. 'de Biol. 74 u. 75.1913.

Weiss, Jahrb. f. Kinderheilk. 35, S. 146. 1893.

Wolff, A., Zieglers Beiträge 28, S. 150. 1900.

- -Eisner, Dermatol. Zentralbl. 1906; zit. nach Bruck.

Wolfsohn, Deutsche med. Wochenschr. 38, S. 1403.1912.

Zappert, Zeitschr. f. klin. Med. 23, S. 227.

Zelenski und Cybulski, Jahrb. f. Kinderheilk. 60, S. 884. 1904.

Zietschmann, Internat. Monatsschr. f. Anat. u. Physiol. 22, S. 89

Zivcovic, Dissertation, Zürich. 1911.

Zollikofer, Deutsches Archiv f. klin. Med. 69, s. 221.

-, Zeitsehr. f. wissenschaftl. Mikroskopie, 1000.

Charlottenburg, Kaiserin Auguste Victoria-Haus. 